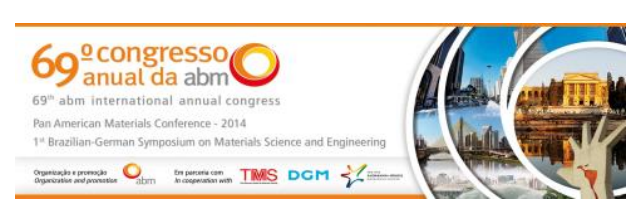

Tema: Gestão de Manutenção

\title{
AUMENTO DA CONFIABILIDADE DAS RETRO ESCAVADEIRAS FROTA 345 DO COMPLEXO MARIANA*
}

Paulinelli Santos Rocha ${ }^{1}$ Cesar David de Paiva² Henrique Rocha Cotta ${ }^{3}$

\section{Resumo}

A frota de equipamentos retro escavadeiras 345 CAT é responsável pela infraestrutura das minas do complexo Mariana, e este Projeto foi desenvolvido devido a baixa confiabilidade que estes equipamentos estavam apresentando. Foi desenvolvido um Projeto de seis sigma para aumentar a confiabilidade do equipamento que é medido pelo indicador MTBF, calculado pela fórmula: horas trabalhadas/ocorrências corretivas. O trabalho foi desenvolvido através de ferramentas de análise e solução de problemas no período de outubro/2012 a agosto/2013. O MTBF médio da frota era 29,39 horas sendo o benchmark de 47 horas. Foi utilizado o método da lacuna para estabelecimento da meta. Após definido a meta foi utilizada ferramentas de priorização dos focos mais relevantes. Para os focos encontrados foram utilizados análise de variabilidade e cartas de proporção, sendo cada causa classificada e priorizada, definido as ações e implantadas. Foi acompanhado o indicador de MTBF e implantados controles de rotina e padronização com o intuito de alcançar o resultado. Por fim, a meta estabelecida foi alcançada, chegando ao MTBF médio da frota de 42,50 horas.

Palavras-chave: MTBF; 345 CAT.

\section{INCREASING THE RELIABILITY OF THE BULLDOZERS 345 FLEET IN MARIANA COMPLEX}

\section{Abstract}

The excavators fleet called CAT 345 is responsible for the Mariana mine site infrastructure and this project was developed due the bad reliability that these equipments experienced in the last Oct/12 to Ago/13. In response was developed Six Sigma Project to increase the reliability measured by the MTBF indicator, the formula is this: work hours divided number of corrective failures. The project was based on analysis tools and solves problems from Oct/12 to Ago/13. The mean MTBF was 29.39 hours with 47 hours regarding benchmark. To establishing the target the "Lacuna methodology" was implemented. After defining the target Priority Tools was used in the Relevant Focus. Under focus found the "Variability analysis" and "Proportion Letters" were used. For each cause classified and prioritized, actions defined, implemented and the MTBF indicator was followed. Routine controls and standardization were implemented in order to achieve the target. In short the target was achieved and the MTBF mean 42.50 hours fleet.

Keywords: MTBF; 345 CAT.

\footnotetext{
Gestor de Recursos Humanos, Técnico mecânico, Vale SA, Mariana, MG, Brasil.

Direito do Trabalho, Técnico mecânico, Vale SA, Mariana, MG, Brasil.

Administrador, Programador na empresa, Vale SA, Mariana, MG, Brasil.
}

\footnotetext{
* Contribuição técnica ao 69ำ Congresso Anual da ABM - Internacional e ao 14ํㅡㄹ ENEMET - Encontro Nacional de Estudantes de Engenharia Metalúrgica, de Materiais e de Minas, 21 a 25 de julho de 2014, São Paulo, SP, Brasil.
} 


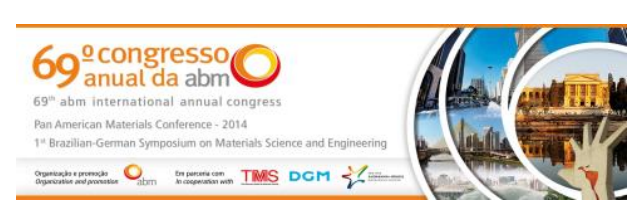

\section{INTRODUÇÃO}

O seis sigma é um programa de excelência que visa à melhoria drástica dos resultados empresariais, busca a redução das ineficiências como falhas, erros, desperdícios, retrabalhos e visa à melhoria. E baseado na aplicação de uma metodologia estruturada e em decisões orientadas por análise de dados e fatos. Possui este nome, pois todos os processos podem ser classificados por um nível sigma de qualidade, esta classificação depende do percentual de defeitos ou não conformidades que estes produzem (MOREIRA, DARÉ, RODRIGUES, SILVA, 2010).

O projeto de seis sigma realizado foi de grande importância para o complexo Mariana e para a empresa Vale, pois a frota 345 CAT é uma frota muito importante para garantir as conformidades nos processos operacionais, sendo esta responsável pela infra estrutura das minas, visto que a mesma não estava apresentando uma confiabilidade no patamar esperado. Pode-se verificar o resultado alcançado foi acima do esperado, o que comprova o sucesso do projeto.

\section{MATERIAIS E MÉTODOS}

Os materiais e métodos utilizados foram orientados através de consultoria realizada pelo Instituto de Desenvolvimento Gerencial (INDG), e finalizado pelo instrutor da (SETA) Desenvolvimento Gerencial. A metodologia utilizada no projeto seis sigma foi o PDCA desdobrado em onze fases conforme pode ser visualizado na Figura 1 abaixo, mas nada impede que se utilize outra metodologia como por ex. DMAIC:

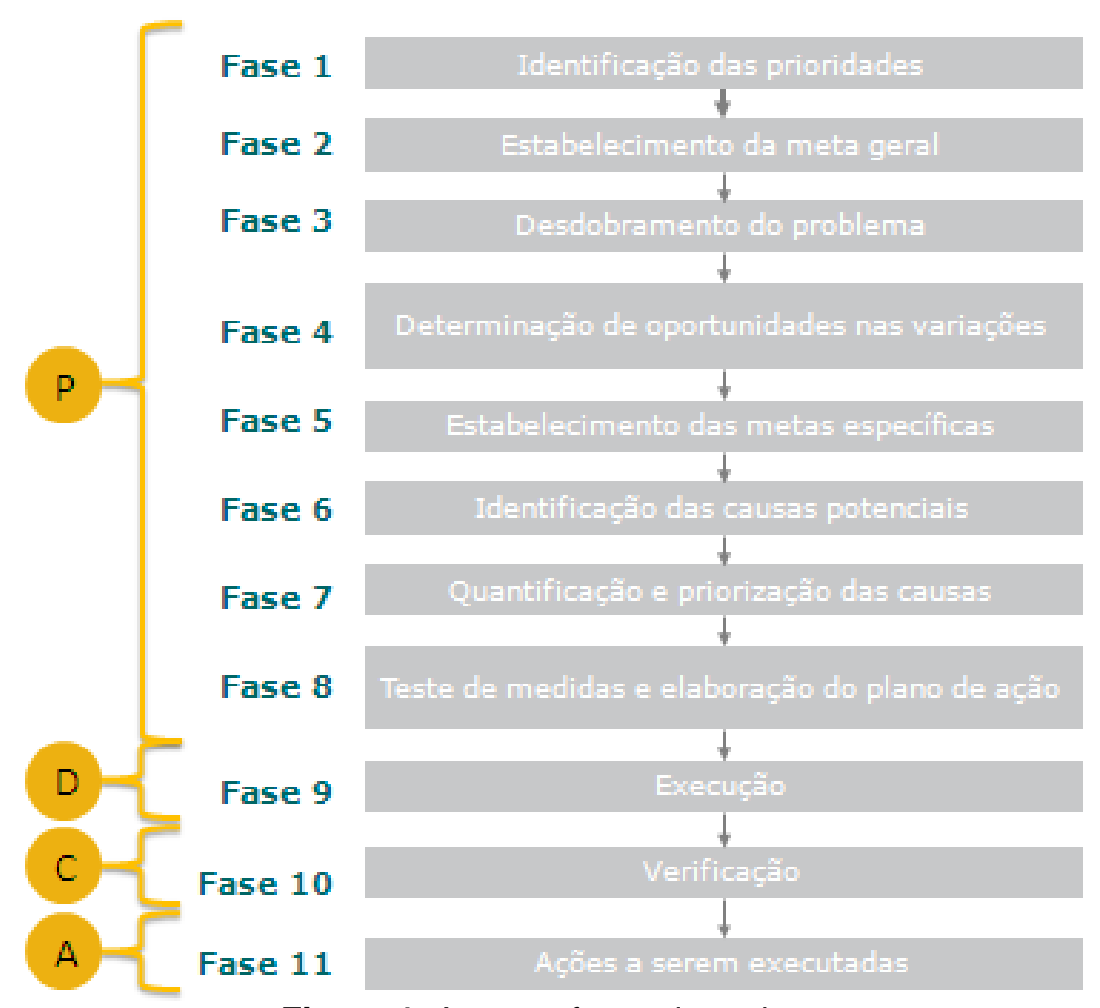

Figura 1. As onze fases do projeto.

As ferramentas utilizadas no projeto em conformidade com o método serão descritas a seguir.

\subsection{Gráfico de Colunas e Linhas}

\footnotetext{
* Contribuição técnica ao $69^{\circ}$ Congresso Anual da ABM - Internacional e ao 14ํㅡㄹ ENEMET - Encontro Nacional de Estudantes de Engenharia Metalúrgica, de Materiais e de Minas, 21 a 25 de julho de 2014, São Paulo, SP, Brasil.
} 
Utilizado na fase I (identificação do problema) para identificar as prioridades o gráfico de linhas, onde apresentou como o indicador se comportava antes do início do projeto. A partir desse gráfico foi traçado a meta geral. Este tipo de gráfico também foi utilizado para apresentar o resultado após a implantação do projeto, com uma média de 42,50 horas de MTBF. Na fase X (verificação) foram utilizados os gráficos de linha e coluna, para verificar o cumprimento das metas específicas.

\subsection{Gráfico Sequencial}

O Gráfico sequencial foi utilizado para analisar o indicador de MTBF nos anos de 2011 e 2012, servindo como base para o início do projeto, e dando continuidade para acompanhamento desse indicador durante e após a implantação do projeto. Foi utilizado nas fases I (identificação do problema) e X (verificação).

\subsection{Perfis de Perdas}

Para o desdobramento do problema (fase III), utilizou-se o perfil de perdas para estratificar as ocorrências de paradas dos sistemas, componentes e itens nos meses de maio, agosto, setembro e outubro do ano de 2012.

\subsection{Diagrama de Árvore}

Foi utilizado para identificar quais são os sistemas, componentes e itens em que havia o maior percentual de perdas (falhas) que afetam de forma negativa para o aumento da confiabilidade da frota e que foi necessário priorizar para o alcance das metas específicas para sustentar a meta geral.

\subsection{Cartas de Proporção}

As cartas de proporção foram utilizadas de forma quantitativa para identificar as causas comuns e especiais de cada um dos sistemas e componentes, com o objetivo de tratá-las.

\subsection{Matriz de Priorização}

Utilizada para priorizar qual o percentual de redução a ser proposto para cada um dos focos priorizados em conjunto com a opinião dos especialistas da área. Foram utilizados os critérios: Criticidade, Facilidade e Autoridade na matriz de priorização (fase V).

\subsection{Diagrama de Causa e Efeito}

O diagrama de causa e efeito foi utilizado na fase VI (identificação das causas potenciais) para cada um dos focos da meta específica, sendo eles: sistema hidráulico, motor, material rodante, sistema elétrico e lubrificação, com o objetivo de encontrar as causas fundamentais de cada um dos focos, a fim de serem tratadas e buscando o alcance do objetivo do projeto.

\subsection{Matriz de Adequabilidade}

* Contribuição técnica ao 69ำ Congresso Anual da ABM - Internacional e ao 14ํㅡㄹ ENET - Encontro Nacional de Estudantes de Engenharia Metalúrgica, de Materiais e de Minas, 21 a 25 de julho de 2014, São Paulo, SP, Brasil. 
Para priorizar as causas de cada foco usamos a matriz de adequabilidade (Fase VII) onde comparamos se a causa levantada (condição atual) era ou não a condição ideal e a provável causa, para assim identificar a causa raiz. Para cada causa raiz utilizamos a matriz de viabilidade para dar sequência.

\subsection{Matriz de Viabilidade}

Utilizamos os critérios: viabilidade técnica, política, econômica, ambiental, econômica e continuidade para verificar se a causa raiz seria ou não tratada.

\subsection{Plano de Ação 5W1H}

Para distribuir as ações utilizados o plano de ação 5W1H (o que, quem, quando, onde, por que, como) (Fase VIII).

\section{RESULTADOS E DISCUSSÃO}

Os resultados alcançados podem ser acompanhados na Figura 2:

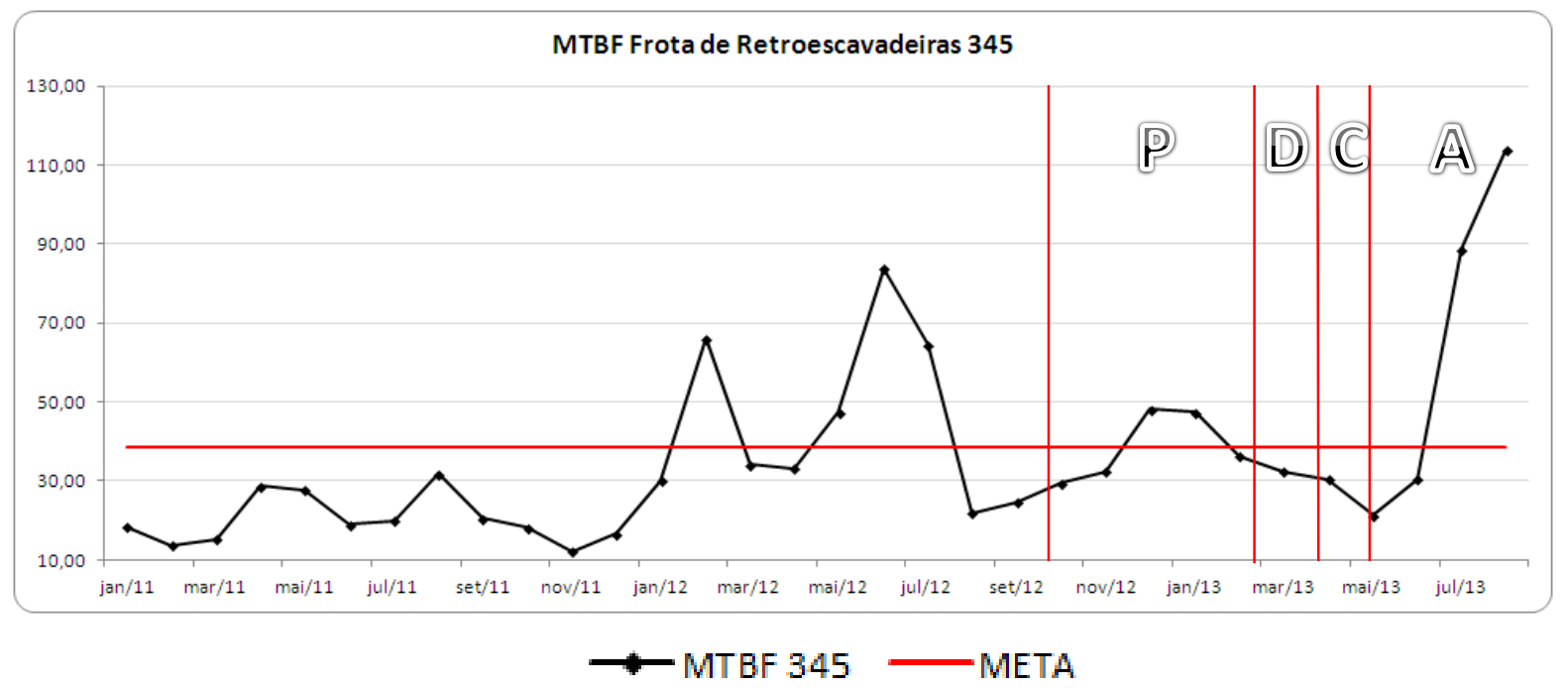

Figura 2. Acompanhamento da meta geral

Pode-se observar que a meta geral foi alcançada ( 42,50 horas), porém no período da execução das ações o indicador estava em queda, após a execução das ações o mesmo ficou acima da meta.

\footnotetext{
* Contribuição técnica ao 69ำ Congresso Anual da ABM - Internacional e ao 14ํㅡㄹ ENEMET - Encontro Nacional de Estudantes de Engenharia Metalúrgica, de Materiais e de Minas, 21 a 25 de julho de 2014, São Paulo, SP, Brasil.
} 

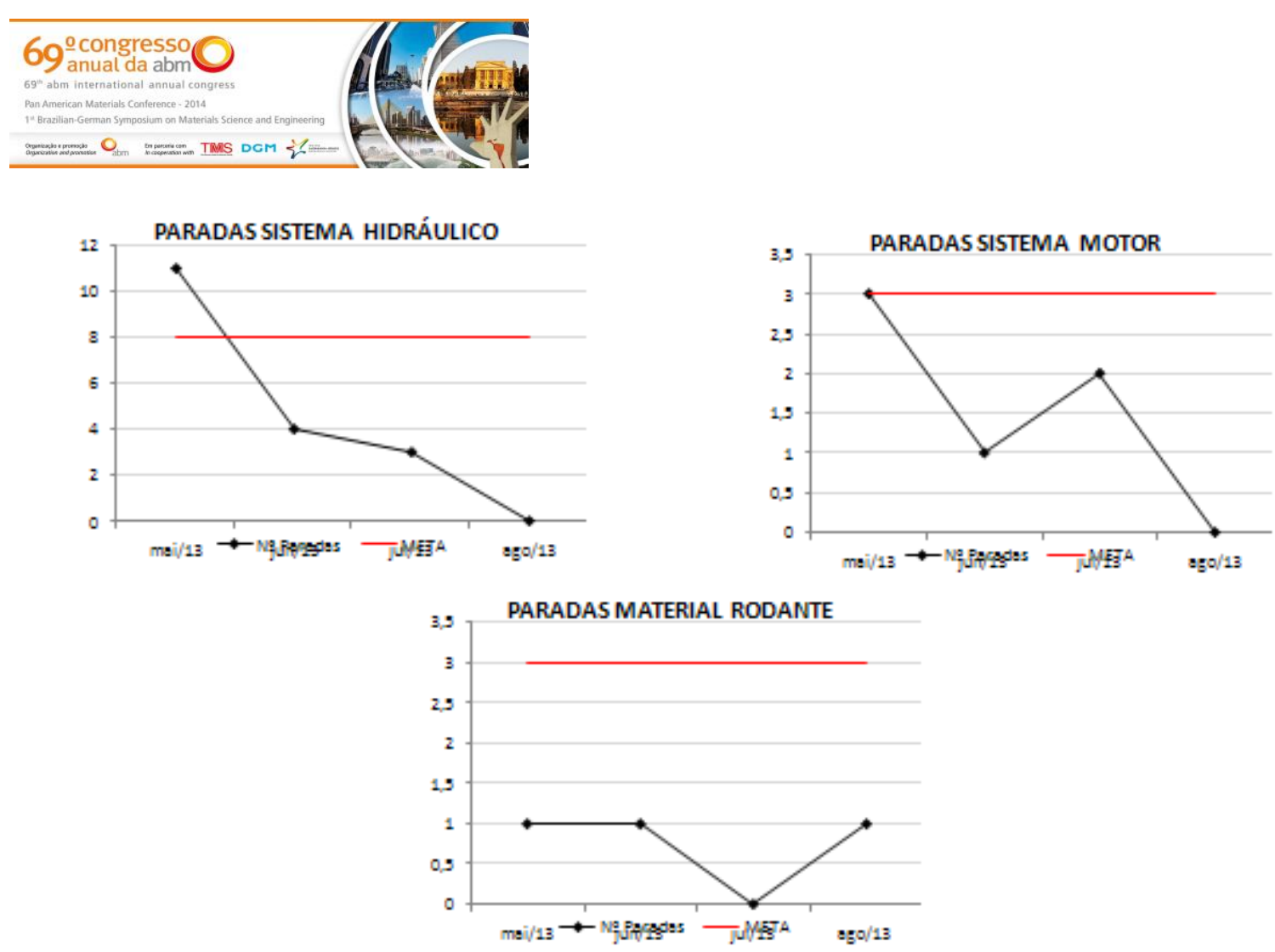

Figura 3. Acompanhamento das metas específicas.

Como apresenta a Figura 3 com o acompanhamento das metas específicas, todas foram alcançadas, contribuindo para o alcance da meta geral.

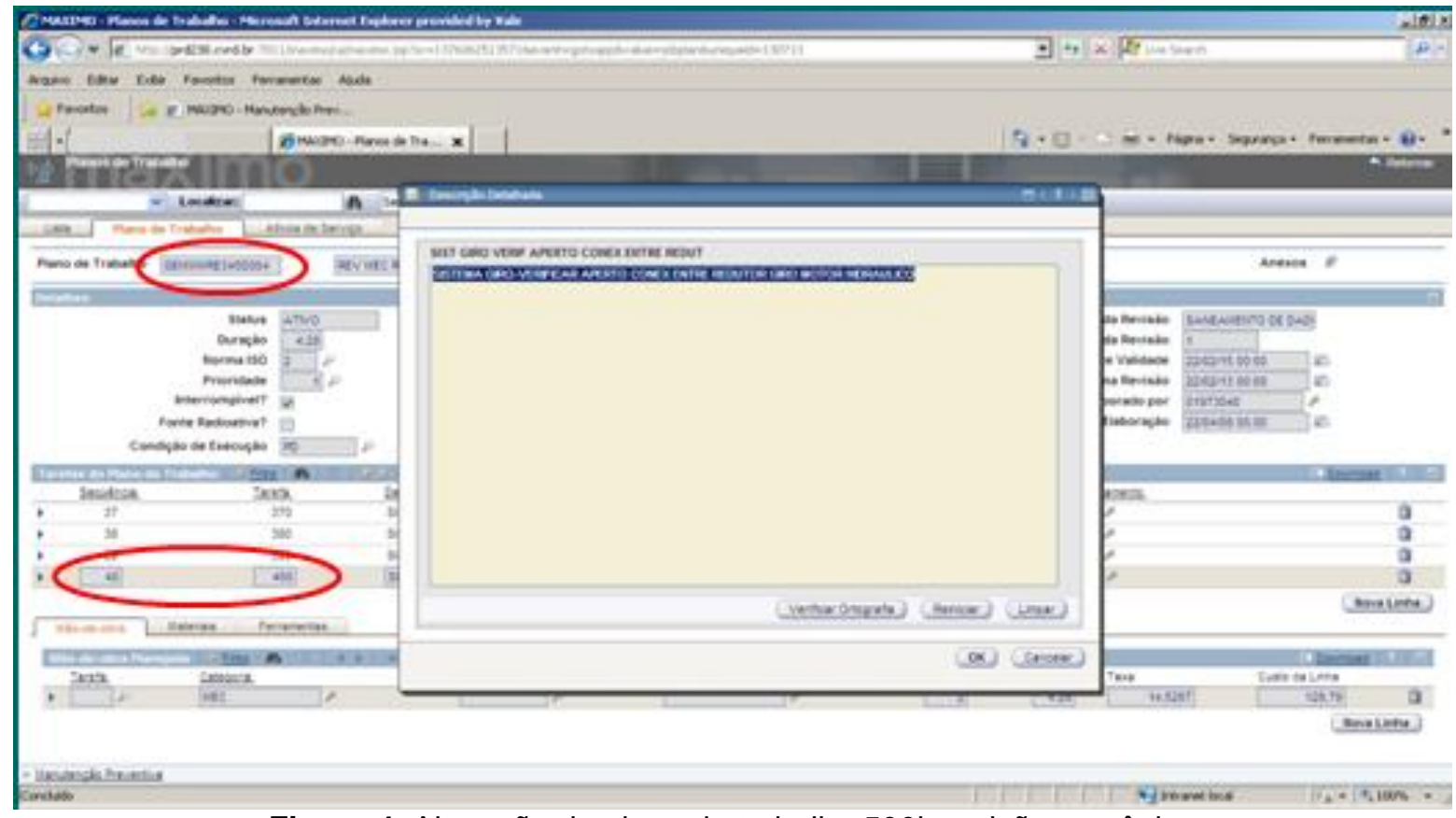

Figura 4. Alteração do plano de trabalho 500h revisão mecânica

* Contribuição técnica ao 69 Congresso Anual da ABM - Internacional e ao 14ํㅡㄹ ENEMET - Encontro Nacional de Estudantes de Engenharia Metalúrgica, de Materiais e de Minas, 21 a 25 de julho de 2014, São Paulo, SP, Brasil. 

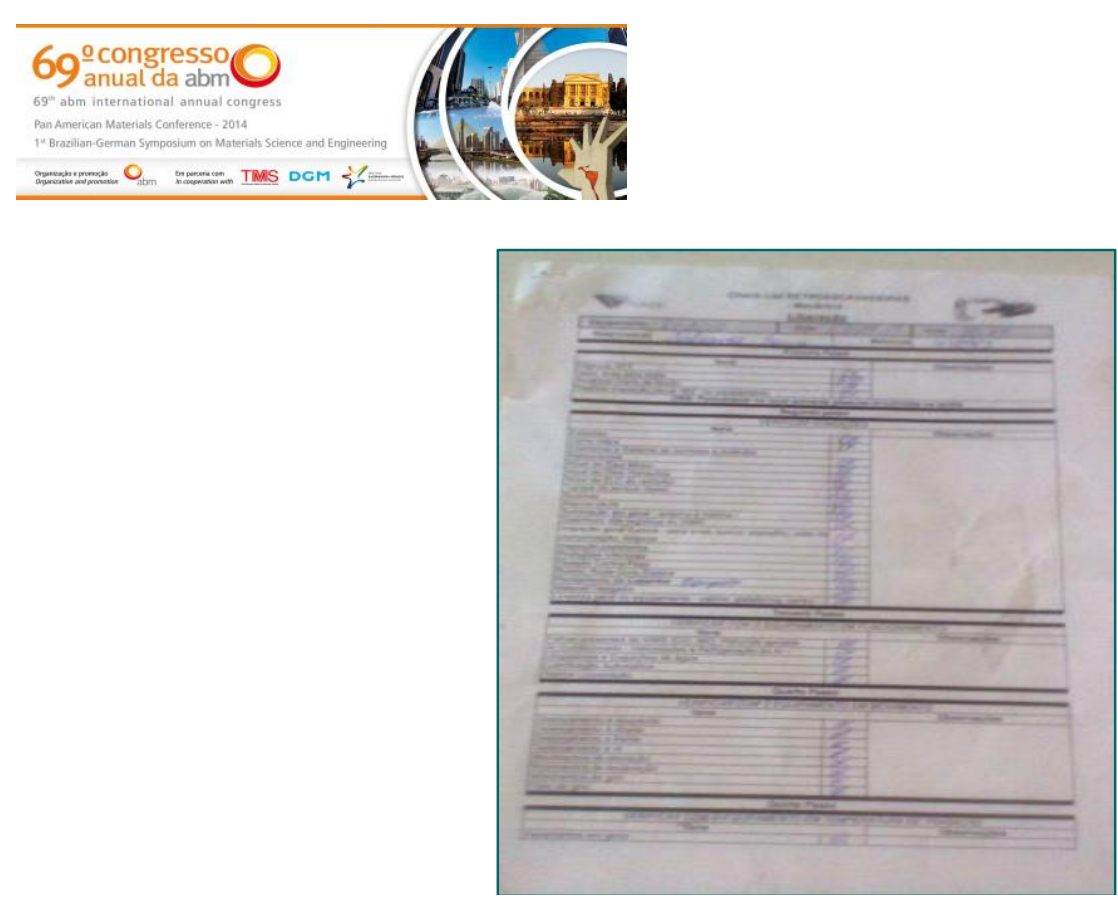

Figura 7: Check list de entrada e saída.

A Figura 8 apresenta o procedimento de tensionamento de esteiras para todos os equipamentos, não somente para a frota 345 , devido ter várias frotas de equipamentos de esteiras. É utilizado para o mecânico consultar a medida correta para o tensionamento da esteira.
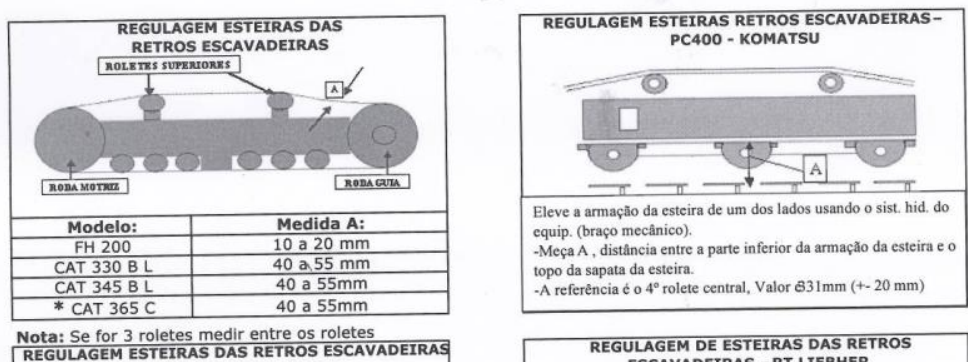

Nota: Se for 3 roletes medir entre os roletes
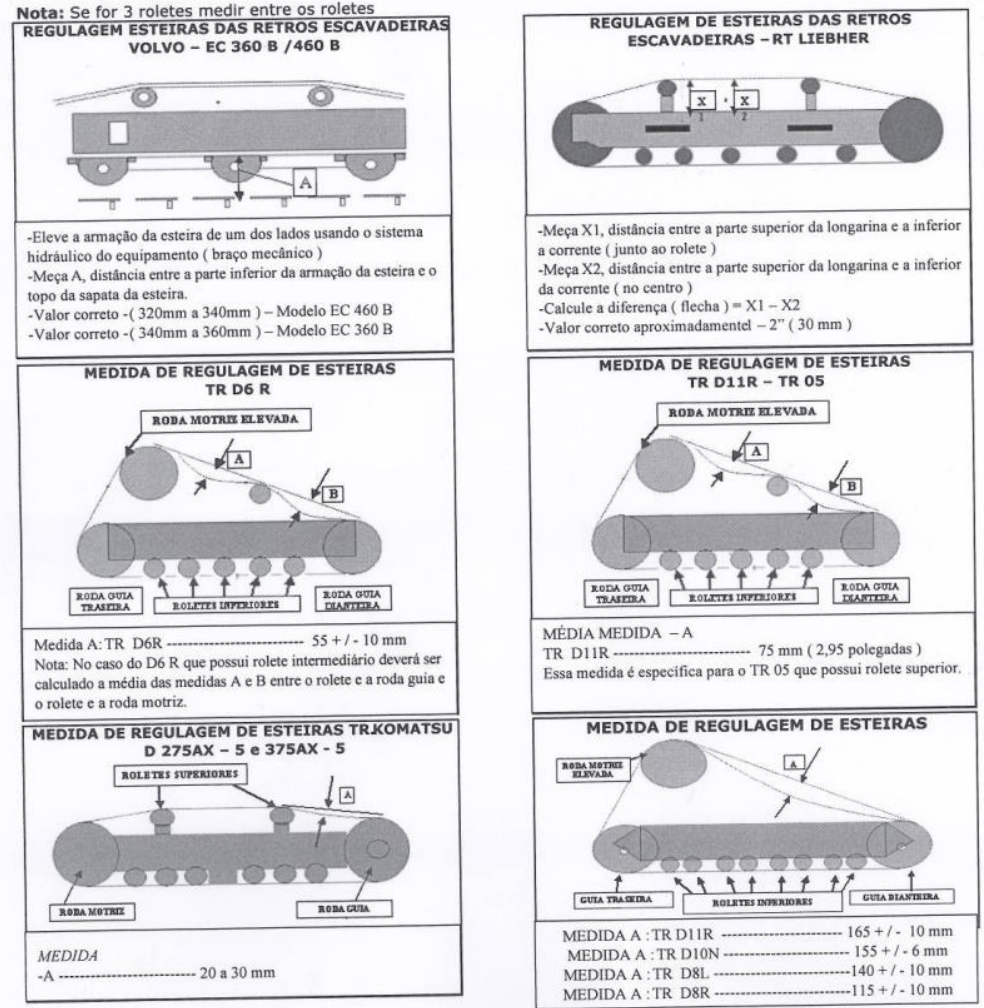

Figura 8. Procedimento para tensionar esteira.

\footnotetext{
* Contribuição técnica ao 69ำ Congresso Anual da ABM - Internacional e ao 14ํㅡㄹ ENEMET - Encontro Nacional de Estudantes de Engenharia Metalúrgica, de Materiais e de Minas, 21 a 25 de julho de 2014, São Paulo, SP, Brasil.
} 


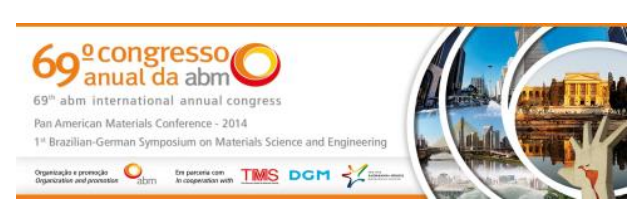

\section{CONCLUSÃo}

A metodologia utilizada foi o PDCA que para este projeto foi desdobrado em onze fases.

As fases de 1 a 8 correspondem à fase $\mathrm{P}$ do PDCA, fase de planejamento, nesta fase definisse qual o problema a ser estudado através, neste caso, do gráfico de colunas e linhas, definição da meta geral com base em histórico (gráfico sequencial), estratificação e focalização do problema (perfil de perdas, diagrama de árvores), avaliação da variabilidade do fenômeno que gera cada problema crítico identificando as oportunidades em cada um dos focos priorizados (cartas de proporção), definição das metas específicas (matriz de priorização), definição das causas potenciais dos focos priorizados (diagrama de causa e efeito), verificação das causas que realmente interferem do projeto (matriz de adequabilidade, matriz de viabilidade) e elaboração do plano de ação $(5 \mathrm{~W} 1 \mathrm{H})$.

Fase 9 corresponde à fase D do PDCA, fase de execução, nesta fase é realizado o acompanhamento das ações propostas e para este fim foi utilizado o plano de ação. Fase 10 corresponde à fase $\mathrm{C}$ do PDCA, fase checar, fase responsável por verificar quanto ao alcance das metas geral e específicas e validação econômica do projeto. As ferramentas utilizadas foram os gráficos sequenciais, gráficos de colunas e linhas. Fase 11 corresponde à fase A do PDCA, fase Agir, esta fase é a responsável por padronizar todas as ações realizadas no projeto para garantir a sustentabilidade do mesmo e para isto foram elaborados procedimentos operacionais e realizado os treinamentos dos envolvidos.

O método utilizado pode ser o PDCA ou o DMAIC, porém as fases sempre serão agrupadas em planejamento, execução, check e ação ou padronização. Já em relação às ferramentas as que aqui foram utilizadas podem variar de projeto para projeto, de autor para autor, cabendo ao usuário utilizar aquela que mais se adeque ao projeto em questão, entretanto a finalidade da utilização de cada uma delas é a mesma independentemente do projeto para cada uma das fases há uma variedade muito grande de ferramentas que podem ser utilizadas.

Independente do tipo de projeto, o objetivo é a busca da melhoria contínua, e para que alcancemos esse objetivo o planejamento é essencial para o sucesso de qualquer trabalho. A utilização da metodologia de forma correta permite o alcance do resultado, mas depende da disciplina e do empenho de toda a equipe, pois por maior que seja o desafio, devemos ter persistência.

\section{BIBLIOGRAFIA}

1 Moreira ACVB, Daré CT, Rodrigues MDF, Silva PA. Programa Seis Sigma Green Belts. Nova Lima: Editora INDG; 2010, versão 8.

\footnotetext{
* Contribuição técnica ao $69^{\circ}$ Congresso Anual da ABM - Internacional e ao 14ํㅡㄹ ENEMET - Encontro Nacional de Estudantes de Engenharia Metalúrgica, de Materiais e de Minas, 21 a 25 de julho de 2014, São Paulo, SP, Brasil.
} 